\title{
Imaging the optic nerve F.W. FITZKE and ganglion cell layer
}

\begin{abstract}
New imaging techniques have extended the limitations of visualisation of the structures of the optic nerve and fundus in the living human eye. Quantitative reconstruction of depth of the optic nerve head is becoming widely available through the use of the confocal scanning laser ophthalmoscope (cSLO). A separate method of quantitative depth reconstruction is possible using stereo pairs which is not subject to the same artefacts as the tomographic

reconstruction. We are investigating the use of these techniques both with conventional stereo imaging and using stereo pairs derived from cSLO images. In these we find that there is additional structural detail evident in the base of the optic nerve head. Further studies made with an instrument optimised to image this region show that this corresponds to the lamina cribrosa. Current work is aimed at further extending the limits of imaging using techniques based on optical coherence tomography, which provides additional depth resolution. Results to date with a prototype device show an improvement of approximately a factor of 10 in depth resolution and that some separate layers of the retina may be visualised including the photoreceptor layer. The goal is to extend these limits to allow visualisation of the ganglion cell layer.
\end{abstract}

Key words Scanning laser ophthalmoscope, Optical coherence tomography, Imaging
New imaging techniques have extended the limits of visualising the living human eye and can provide quantitative measurements of fundus features. One of the most important developments has been based on the scanning laser ophthalmoscope (SLO), which has the property of providing optical sections through the use of confocal optics. ${ }^{1}$ This can reveal structures which would otherwise be invisible due to the contrast-degrading effects of overlying elements, and can use tomographic reconstruction methods to provide quantitative depth information. These developments have had a major impact on ophthalmology and continue to advance the limits of seeing and measuring properties of the eye. The depth resolution of commonly used SLOs can be of the order of several hundred micrometres. In this way small vessels or other structural detail obscured by the scattering of neural tissue in the optic nerve can be seen and some of the contrast-degrading effects of ocular media opacities can be bypassed. New opportunities continue to appear so that we now are able to see and quantify the structure of the lamina cribrosa using a prototype SLO. ${ }^{2-5}$

One of the most widely used techniques with the SLO is the quanitification of depth information of the optic nerve head using tomographic reconstruction. However, there a number of limitations and artefacts which can arise and so an independent means of quantifying depth information is desirable. This can be achieved through quantitative stereo
F.W. Fitzke

Department of Visual Science Institute of Ophthalmology University College London Bath Street London EC1V 9EL, UK

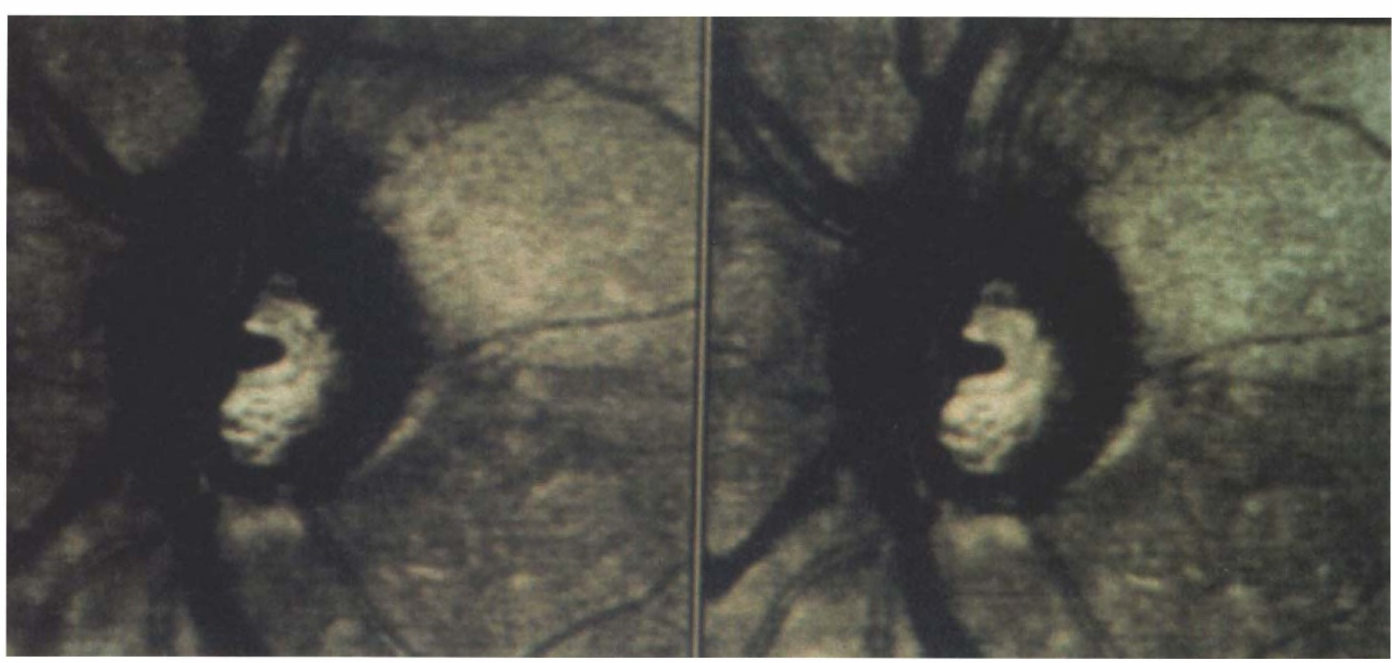

Fig. 1. Stereo pair of images taken with a Rodenstock scanning laser ophthalmoscope. Each image is the average of 32 individual images which have been averaged after correction for eye movements. 


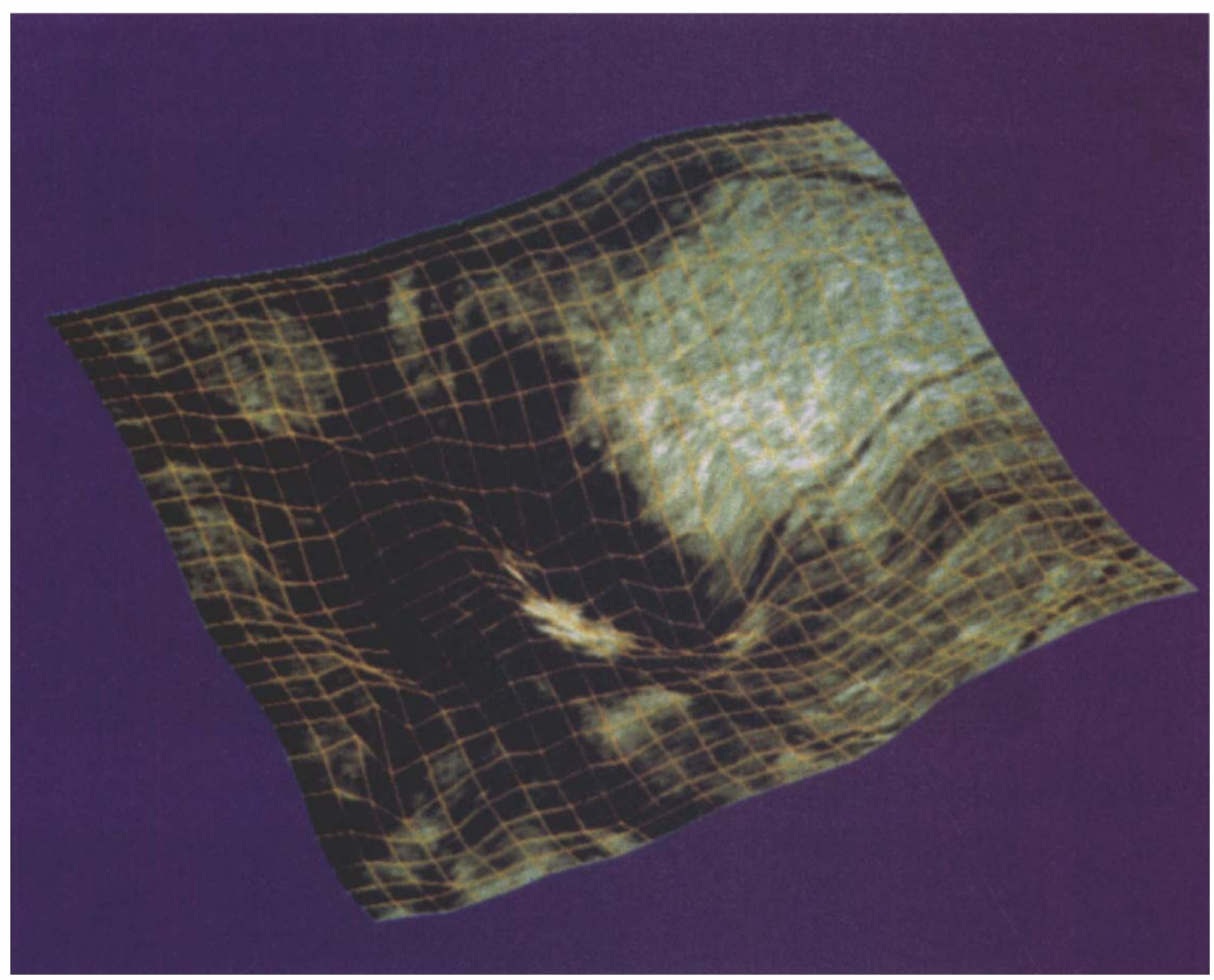

Fig. 2. A reconstructed depth map from a stereo pair using disparity calculation.

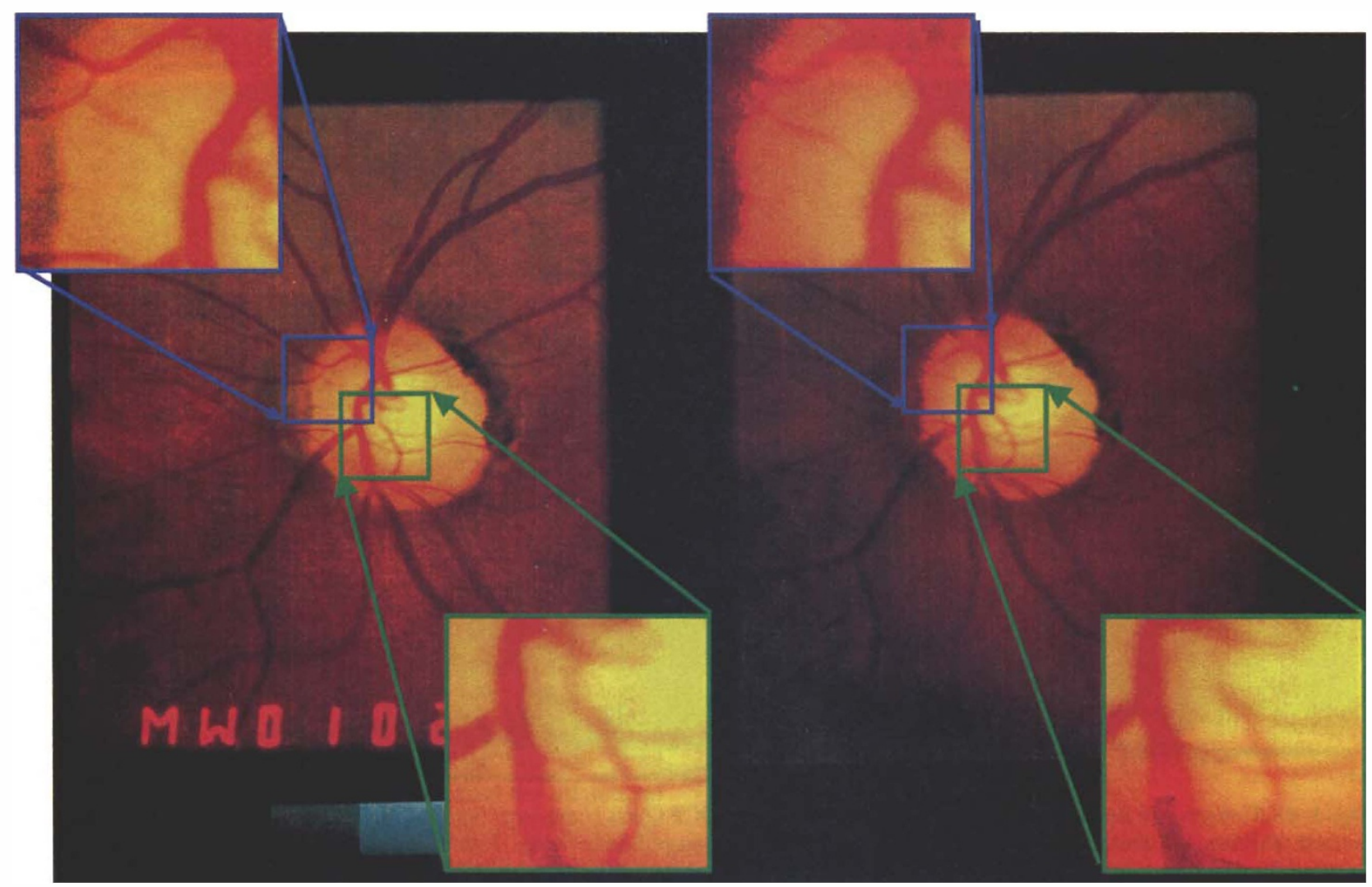

Fig. 3. Example of calculation of depth from stereo pairs. For each of the image pairs, two windows are illustrated as outlined in green and blue, as well as the magnified views of each. For the region outlined in blue on the left, the corresponding pattern match on the right is shown, also in blue. Similarly the corresponding pattern match for the other location is shown as outlined in green. The process is repeated on a matrix of 10310 locations on the optic nerve head and the resulting disparities used to calculate depth as seen in Fig. 2. 


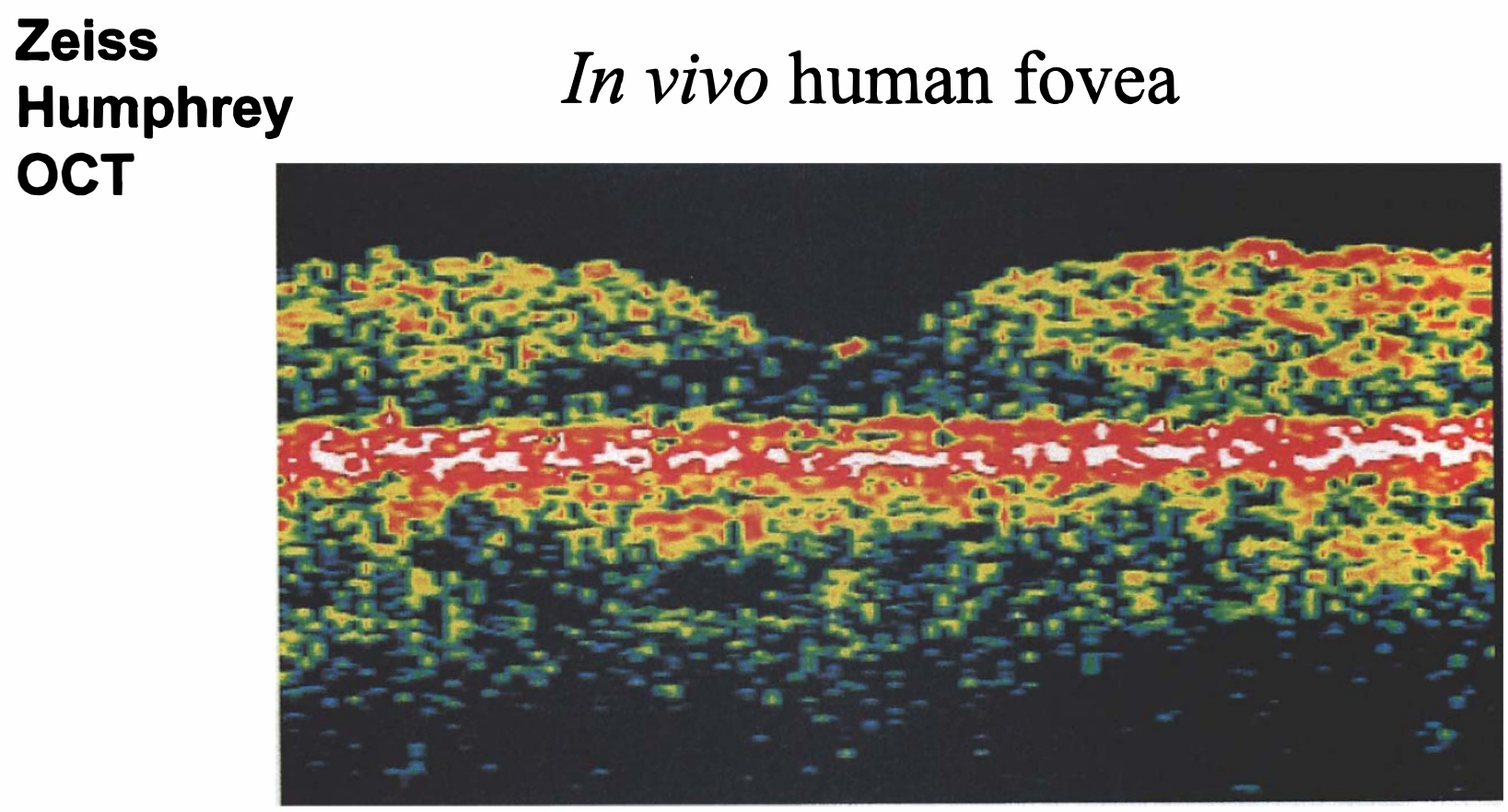

\section{Prototype High Magnification OCT}
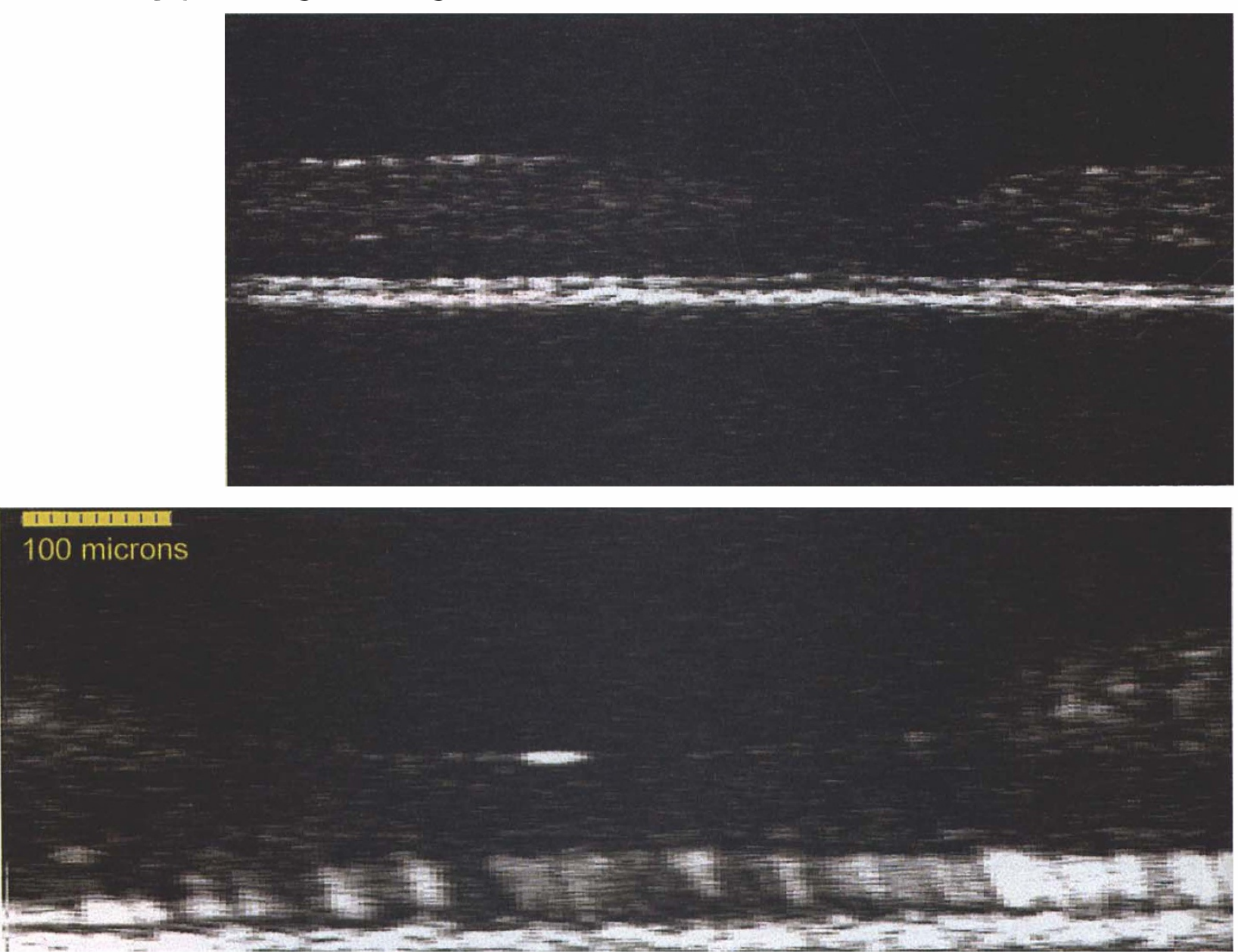

Fig. 4. OCT images of a normal fovea from a commercially available OCT (Zeiss Humphrey) (top) and from a new prototype device (middle and bottom). The foveal pit can be seen in all three images, and in the high-magnification image (bottom), where the foveal pit extends over the entire width of the image, vertically oriented structures corresponding to the photoreceptor layer are visible. Modified from Podoleanu et al. ${ }^{18}$

reconstruction from stereo pairs of images. We have investigated this potential both with conventional stereo pairs and with those derived from SLO stereo pairs. An example is shown in Fig. 1. This shows a stereo pair of images taken with a Rodenstock SLO where the separation between pairs of images in the pupil was set 
to $3 \mathrm{~mm}$. Each pair of images is the average of 32 individual images which were aligned to compensate for eye movements and then averaged to reduce noise and improve contrast. From these stereo pairs depth information was deduced and then plotted as a wire frame diagram over the original image as illustrated in Fig. 2. Here it can be seen that the quantitative depth information describes the surface of the cup of the optic nerve head as expected.

The method used to calculate depth differs from that used in conventional SLO imaging in that it was derived from disparity calculations between the pairs of images rather than using tomographic methods. This has the advantage of not being subject to some of the artefacts inherent in tomographic reconstructions, although there are other optical considerations which must be taken into account. The method is illustrated in Fig. 3, in this case using a stereo pair from a Nidek stereo fundus camera. For each small region within the image the corresponding best pattern match is automatically found by the computer and the horizontal displacement of each pattern used to calculate disparity and depth.

In developing these new techniques we found that in these high-resolution SLO images additional structural features within the optic nerve head became visible. These were further investigated and found to be attributable to the pores of the lamina cribrosa. These are visible in Fig. 1. The lamina cribrosa is of interest in glaucoma since it is through this structure that the nerve fibres pass from the retina to the lateral geniculate nucleus, and it has been implicated in the mechanism of damage in glaucoma. ${ }^{2,3,6-13}$

We have imaged the structure of the pores of the lamina cribrosa and measured visual function psychophysically in the same eyes of patients with glaucoma. ${ }^{2,3}$ We found that the degree of visual field loss was associated with abnormalities in the shapes of the pores. Further work is under way to improve the resolution of this structure and its changes with depth in the living human eye.

Recently a technique based on optical coherence tomography (OCT) has been developed. ${ }^{14-17}$ This further extends depth resolution and has promise for measuring the thickness of different retinal layers. We have developed a prototype device based on the OCT technique that gives high-magnification images of the retina in vivo. ${ }^{18}$ An example is shown in Fig. 4 . This has provided higher-magnification images than have previously been possible and in images of the foveal region the separate structural details of the retina are becoming visible.

The goal of current work is to further extend the limits of imaging in the living human eye so that the structural changes can be visualised for the different cell layers of the retina. This will allow the investigation in patients with glaucoma of the basis of the damage in such structures as the lamina cribrosa and the neural retina, in comparison with the psychophysically measured losses of visual function.

\section{References}

1. Webb RH, Hughes GW. Scanning laser ophthalmoscope. IEEE Trans Biomed Eng 1981;28:488-92.

2. Bhandari A, Fontana L, Fitzke FW, Hitchings RA. Quantitative analysis of the lamina cribrosa in vivo using a scanning laser ophthalmoscope. Curr Eye Res 1997;16:1-8.

3. Fontana L, Bhandari A, Fitzke FW, Hitchings RA. In vivo morphometry of the lamina cribrosa and its relation to visual field loss in glaucoma. Curr Eye Res 1998;17:363-9.

4. Fitzke FW, Masters BR. Three-dimensional visualization of confocal sections of in vivo human fundus and optic nerve. Curr Eye Res 1993;12:1015-8.

5. Woon WH, Fitzke FW, Bird AC, Marshall J. Confocal imaging of the fundus using a scanning laser ophthalmoscope. Br J Ophthalmol 1992;76:470-4.

6. Birch M, Brotchie D, Roberts N, Grierson I. The threedimensional structure of the connective tissue in the lamina cribrosa of the human optic nerve head. Ophthalmologica 1997;211:183-91.

7. Dandona L, Quigley HA, Brown AE, Enger C. Quantitative regional structure of the normal human lamina cribrosa: a racial comparison. Arch Ophthalmol 1990;108:393-8.

8. Elkington AR, Inman CB, Steart PV, Weller RO. The structure of the lamina cribrosa of the human eye: an immunocytochemical and electron microscopical study. Eye 1990;4:42-57.

9. Jonas JB, Mardin CY, Scholtzer-Schrehardt U, Naumann GO. Morphometry of the human lamina cribrosa surface. Invest Ophthalmol Vis Sci 1991;32:401-5.

10. Miglior S, Rossetti L, Lonati C, Orzalesi N. Scanning laser ophthalmoscopy of the optic disc at the level of the lamina cribrosa. Curr Eye Res 1998;17:453-61.

11. Quigley H, Pease ME, Thibault D. Change in the appearance of elastin in the lamina cribrosa of glaucomatous optic nerve heads. Graefes Arch Clin Exp Ophthalmol 1994;232:257-61.

12. Albon J, Karwatowski WS, Avery N, Easty DL, Duance VC. Changes in the collagenous matrix of the aging human lamina cribrosa. Br J Ophthalmol 1995;79:368-75.

13. Yan DB, Coloma FM, Metheetrairut A, Trope GE, Heathcote JG, Ethier CR. Deformation of the lamina cribrosa by elevated intraocular pressure. Br J Ophthalmol 1994;78:643-8.

14. Fujimoto JG, Brezinski ME, Tearney GJ, Boppart SA, Bouma $B$, Hee MR, et al. Optical biopsy and imaging using optical coherence tomography. Nature Med 1995;1:970-2.

15. Gurses-Ozden R, Ishikawa H, Hoh ST, Liebmann JM, Mistlberger A, Greenfield DS, et al. Increasing sampling density improves reproducibility of optical coherence tomography measurements [in process citation]. J Glaucoma 1999;8:238-41.

16. Pieroth L, Schuman JS, Hertzmark E, Hee MR, Wilkins JR, Coker J, et al. Evaluation of focal defects of the nerve fiber layer using optical coherence tomography. Ophthalmology 1999;106:570-9.

17. Schuman JS, Pedut-Kloizman T, Hertzmark E, Hee MR, Wilkins JR, Coker JG, et al. Reproducibility of nerve fiber layer thickness measurements using optical coherence tomography [see comments]. Ophthalmology 1996;103:1889-98.

18. Podoleanu AG, Seeger M, Dobre GM, Webb DJ, Jackson DA, Fitzke FW. Transverse and longitudinal images from the retina of the living eye using low coherence reflectometry. J Biomed Optics 1998;3:12-20. 